\title{
Calpain-10 drives podocyte apoptosis and renal injury in diabetic nephropathy
}

This article was published in the following Dove Press journal:

Diabetes, Metabolic Syndrome and Obesity: Targets and Therapy

\author{
Tao Wang' \\ Yanbin Gao (D) ${ }^{1,2}$ \\ Xiaolei Wang' \\ Yimin Shi ${ }^{1}$ \\ Jiayi $\mathrm{Xu}^{\prime}$ \\ Bingjie $\mathrm{Wu}^{\prime}$ \\ Jiaxin $\mathrm{He}^{\mathrm{I}}$ \\ Yimeng $\mathrm{Li}^{2}$
}

'School of Traditional Chinese Medicine, Capital Medical University, Beijing, People's Republic of China; ${ }^{2}$ Beijing Key Laboratory of TCM Collateral Disease Theory Research, Beijing, People's Republic of China
Correspondence: Yanbin Gao School of Traditional Chinese Medicine, Capital Medical University, No. I0, Youanmenwai, Xitoutiao, Fengtai District, Beijing 100069, People's Republic of China $\mathrm{Tel}+86108391 \quad 1720$

Email dfyynfm@163.com
Background: Diabetic nephropathy (DN) is a progressive microvascular complication of diabetes mellitus (DM), driven largely by podocyte apoptosis. The cysteine protease Calpain 10 is known to augment apoptosis and necrosis, and is a potential therapeutic target in DN. Methods: Type 2 diabetes was induced in SD rats by high-fat diet (HFD) feeding and streptozotocin (STZ) injections, and simulated in vitro by culturing conditionally immortalized mouse podocytes in hyperlipidemic (PA, $100 \mu \mathrm{M})$ conditions. The rate of apoptosis in the renal tissues and cultured podocytes was determined by TUNEL assay. The expression of Calpain 10 and its biological effects were assayed by real-time PCR, Western blotting, immunofluorescence and electron microscopy.

Results: Calpain 10 was up-regulated in the kidneys of DN rats, as well as immortalized mouse podocytes. High levels of Calpain 10 was associated with renal dysfunction and tissue destruction, and podocyte injury and apoptosis. Knockdown of Calpain 10 protected podocytes by decreasing apoptosis rate, and upregulated nephrin.

Conclusion: Calpain 10 is a pro-apoptotic factor in $\mathrm{DN}$, and can be targeted for treating glomerular diseases.

Keywords: Calpain 10, podocyte, apoptosis, diabetic nephropathy

\section{Introduction}

Diabetic nephropathy (DN), a progressive microvascular complication of diabetes mellitus (DM), is the primary reason for end-stage renal diseases (ESRD). ${ }^{1}$ The onset and progression of DN is accompanied by dysfunctional podocytes, ${ }^{2-4}$ the specialized cells that are present around the glomerular capillaries and form the filtration barrier. Podocyte injury plays a key role in the increased microalbuminuria seen in DN, which is also characterized by a significant loss in podocyte numbers due to apoptosis. ${ }^{5,6}$ Therefore, inhibiting podocyte apoptosis is a potential therapeutic strategy in DN. One possible way to induce apoptosis and necrosis is through Calpain10 which is a mitochondrial and cytosolic $\mathrm{Ca}^{2+}$-regulated cysteine protease. ${ }^{7,8}$ A genome-wide linkage scan for diabetes susceptibility genes revealed that Calpain 10 was associated with an increased risk of type 2 DM. ${ }^{9,10}$ It plays an important role in insulin secretion, glucose uptake in skeletal muscle and other tissues, mitochondrial respiration. The aim of this study was to determine the possible role of Calpain 10 in hyperlipidemia-induced podocyte injury and renal dysfunction.

\section{Materials and methods}

\section{Reagents}

Mouse monoclonal anti-nephrin antibody (Cat\#:sc-377246, LOT: G0213) was purchased from Santa Cruz Biotechnology, Inc. (CA, USA). Rabbit polyclonal anti-Calpain 10 
antibodies (Cat\#: ab28226, LOT: GR342802-7) were purchased from Abcam (Cambridge, UK). Rabbit polyclonal anti-caspase-3 antibodies (Cat\#: 9662s, LOT: 18) were purchased from Cell Signaling Technology, Inc. (Danvers, MA, USA). GAPDH mouse monoclonal antibodies (Cat\#:60004-1lg, LOT:10003343) were purchased from Prointech Group (Rosemont, USA). HRP goat anti-rabbit IgG (Cat\#:RS0002, LOT: B0201) and HRP goat anti-mouse IgG (Cat\#:RS0001, LOT: B0101) were purchased from ImmunoWay Biotechnology (TX, USA). Rhodamine (TRITC)-Conjugated Goat anti-Mouse IgG(H+L) (Cat\#: ZF-0313, LOT:135850) and Fluorescein-Conjugated Goat anti-Rabbit $\operatorname{IgG}(\mathrm{H}+\mathrm{L})$ (Cat\#: ZF-0311, LOT:136851) were purchased from ZSGBBIO (Beijing, China).

\section{Cell culture}

A conditionally immortalized mouse podocyte cell line (BNCC337685) was obtained from $\mathrm{BeNa}$ Culture Collection (Beijing, China). The cells were cultured in DMEM/low glucose medium (Genview, Florida, USA) supplemented with $10 \%$ fetal bovine serum (FBS) (Gibco, Carlsbad, CA, USA) and recombinant IFN- $\gamma$ (PeproTech, London, UK) at $33{ }^{\circ} \mathrm{C}$ for proliferation, and without IFN- $\gamma$ at $37{ }^{\circ} \mathrm{C}$ for more than 7 days for differentiation. Once they reached $\sim 80 \%$ confluency, the podocytes were maintained in serum-free conditions for $24 \mathrm{~h}$ and then used for various analyses.

\section{Proliferation assays}

The podocytes were seeded in 96-well plates with serumfree DMEM at the density of $8 \times 10^{4}$ per well, and cultured for $24 \mathrm{~h}$, followed by induction with 50,100 and $150 \mu \mathrm{M}$ palmitate (PA) (Sigma-Aldrich, St. Louis, MO, USA) for $24 \mathrm{~h}, 48 \mathrm{~h}$ or $72 \mathrm{~h}$. The suitably treated cells were then incubated for another $2 \mathrm{~h}$ with cell-counting kit-8 (CCK-8, KeyGEN BioTECH, Nanjing, China) solution (10 $\mu \mathrm{L} /$ well). The optical density of each well was measured at $450 \mathrm{~nm}$. Taking the viability of normal podocytes as $100 \%$, a decrease to $30 \%$ was considered an injured podocyte model. $^{11,12}$

\section{Small interfering RNA (siRNA) transfection}

Both the Calpain 10-specific siRNA (siCalpian 10) and scrambled siRNA (Calpian10-Con) were designed and synthesized by RiboBio Co., Ltd. The podocytes were transfected according to the manufacturer's protocol, and analyzed for
Calpain 10 levels $48 \mathrm{~h}$ later. The siRNA sequence targeting Calpain 10 was GCAGAAAGGTGGAGCTTGA.

\section{Establishment of DN model}

A total of 60 male Sprague-Dawley rats (8 weeks old, weighing 200-250 g), were randomized into the control group (Control, $\mathrm{n}=15$ ) and the $\mathrm{DN}$ model group (DN, $\mathrm{n}=45)$. The control group was injected with an equal volume of the vehicle $(0.1 \mathrm{M}$ citrate buffer, $\mathrm{pH} 4.5)$ as the placebo/normal control and fed a normal diet. The latter was intravenously injected once with STZ (Sigma-Aldrich, St. Louis, MO, USA) in sodium citrate buffer $(55 \mathrm{mg} / \mathrm{kg}$ body weight) through caudal vein and fed with the high fat diet (HFD; $10 \%$ lard, 20\% sucrose, $2.5 \%$ cholesterol, $0.5 \%$ sodium cholate and $67 \%$ basic feed) to induce type 2 diabetes. ${ }^{13-15}$ Random blood glucose (RBG) levels were measured in blood draw from the caudal vein of each animal with a portable glucometer (One Touch ${ }^{\circledR}$ Ultra, LifeScan, Johnson \& Johnson, CA, USA). After 1 week, the rats with $\geq 16.7 \mathrm{mmol} / \mathrm{L}$ fasting blood glucose for 3 consecutive days was recognized as diabetic ones; otherwise, they were excluded from the study. ${ }^{16-18}$ After establishing the diabetes, the DN group rats were continued to be fed with high fat diet to induce diabetic nephropathy, ${ }^{19}$ and further the DN group rats were euthanized at 3 time points (week 4, 8, and 12 post confirming the diabetes). The blood samples were collected to detect the level of blood serum creatinine ( $\mathrm{SCr}$ ), total cholesterol (TC) and triglyceride (TG), and the urine was collected to measure albumin levels, and urine albumin creatinine ratio (UACR); the fresh kidneys were dissected and processed for hematoxylin-eosin (HE), periodic acid Schiff (PAS) and Masson's stainings as per standard protocols. The alterations of renal histopathology combined with increased UACR indicated successful establishment of the DN model, otherwise, they were excluded. All animal experiments were approved by the Institutional Animal Care and Use Committee at Capital Medical University, and conformed to the Guidelines for the Care and Use of Laboratory Animals by the National Institute of Health.

\section{Morphological studies}

Fresh kidneys from each rat were fixed in 4\% paraformaldehyde (KeyGEN BioTECH, Nanjing, China), and embedded in paraffin. Then 4-6 $\mu$ m-thick sections were transferred to glass slides, and processed for HE, PAS and Masson's staining. Morphologic alterations were examined by light microscopy (Leica DM60008, Kyoto, Japan). 
Sections of the renal cortex were harvested and fixed in $2 \%$ glutaraldehyde (Servicebio, Wuhan, China), and ultrathin sections were then collected for staining. ${ }^{16}$ The samples were observed with a transmission electron microscope (EM, JEM-1400 plus, JEOL, Tokyo, Japan).

\section{Detection of markers relevant to renal function and serum lipid level}

Serum total cholesterol and triglycerides were assayed using reagent kits purchased from Prodia Diagnostics (Botzingen, Germany) according to the experimental protocol. Serum creatinine, urine creatinine and urine albumin were determined using related kits purchased from Leadman Biochemistry (Beijing, China) in accordance with manufacturer's instructions. All the biochemical parameters were measured with an automatic chemistry analyzer (7600, HITACHI, Tokyo, Japan). The urinary protein content was calculated in terms of the creatinine concentration (urinary protein/urinary creatinine).

\section{Immunofluorescence (IF)}

The suitably processed kidney sections were blocked with $10 \%$ goat serum (ZSGB-BIO, Beijing, China) for $1 \mathrm{hr}$, and then incubated overnight with rabbit anti-nephrin (1:50) and rabbit anti-Calpain 10 (1:200) antibodies at $4{ }^{\circ} \mathrm{C}$. After incubation with the corresponding secondary antibody (1:100) for $1 \mathrm{hr}$ at $37^{\circ} \mathrm{C}$, the sections were counterstained with 4', 6'-diamidino-2-phenylindole (DAPI) (Genview, Florida, USA), and imaged using a laser confocal microscope (TCS SP8 STED, Leica, Wetzlar, Germany).

\section{Western blotting}

The total protein of cultured podocytes and kidney tissues were extracted, and equal amount of proteins (20 g/lane) per sample were separated by $10 \%$ SDS-PAGE and transferred to PVDF membrane. The latter was blocked with $5 \%$ skimmed milk in PBS $+0.05 \%$ tween 20 , followed by overnight incubation with the anti-Calpain 10 (1:1000), anti-nephrin (1:500) and anti-caspase 3 primary antibodies $(1: 1000)$ at $4{ }^{\circ} \mathrm{C}$. After incubating the blots with corresponding peroxidase-conjugated secondary antibody (1:5000), the positive bands were developed using a chemiluminescent reagent (Thermo Scientific, USA).

\section{Quantitative RT-PCR}

Total RNA was isolated from cultured podocytes and the renal cortex using TRIzol Reagent according to the manufacturer's instructions, and reverse transcribed using the PrimeScript ${ }^{\mathrm{TM}}$ RT kit. The $\mathrm{qPCR}$ analysis was performed using the SYBR ${ }^{\circledR}$ Premix Ex Taq ${ }^{\mathrm{TM}}$ II (Takara) in the ABI PRISM 7500 FAST Real-TIME PCR System (ABI, Vernon, CA, USA). The relative expression of Calpain-10 mRNAs were calculated using the $2^{-\Delta \Delta C t}$ method.

\section{TUNEL assay}

The rate of apoptosis in cultured podocytes and kidney tissues was measured using the In Situ Cell Death Detection kit (Nanjing KeyGen Biotech Co., Ltd, China) according to the manufacturer's instructions. Tissue sections and podocytes cultured on coverslips were treated with proteinase $\mathrm{K}(20 \mathrm{~g} / \mathrm{mL})$, and stained as recommended. The apoptotic index of kidneys was evaluated in terms of the TUNEL positive cells selected randomly from 10 fields at $200 \times$ magnification; and the apoptotic podocytes was quantitatively analyzed by counting the TUNEL-positive cells in 10 non-overlapping fields for each treatment group under $200 \times$ magnification. Results were presented as the number of TUNEL-positive cells per $10^{3}$ cells and \%TUNEL-positive podocytes.

\section{Statistical analysis}

All data are presented as mean \pm standard deviation (SD). Statistical analysis was performed using the SPSS 19.0 software (IBM Corporation, Armonk, NY, USA). Student's $t$-test and one-way ANOVA were respectively used to compare two and multiple groups. Spearman's rank correlation coefficients were evaluated to access the relationships between the level of Calpain 10 and UACR. A $P$-value $<0.05$ was considered statistically significant.

\section{Results \\ Calpain 10 was upregulated in diabetic rats and cultured podocytes}

The biochemical parameters of the studied groups were shown in Figure 1A-E. In week 0, $\mathrm{FB} \geq 16.7 \mathrm{mmol} / \mathrm{L}$ in the $\mathrm{DN}$ group was indicative of diabetes. To ensure $\mathrm{DN}$ rat model established successfully, Scr and UACR was measured. Compared to the control group, the levels of Scr and UACR in DN group showed no significant difference at 0 week; then remarkably increased by the 4 th week after establishing diabetes which means DN group rats emerged renal insufficiency and continuously climbing as the modeling time prolonged (Figure 1D, E). Renal tissues were 

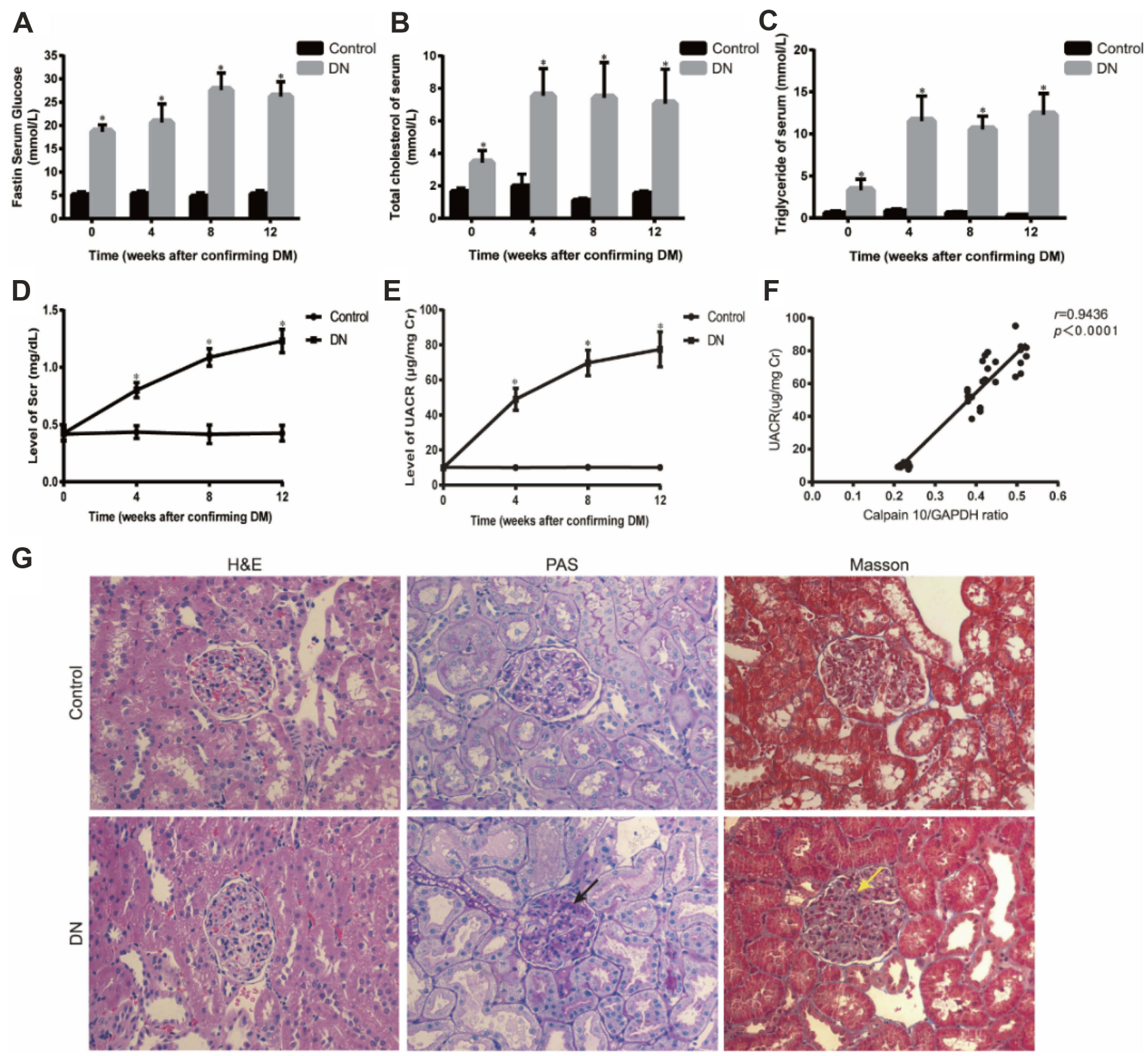

Figure I Establishment of diabetic nephropathy in rats. (A-E) Physical and biochemical parameter of experimental animals showed that DN rats emerged hyperglycemia, hyperlipemia and renal insufficiency. (F) Correlation between Calpain I 0 and UACR. (G) Renal histopathology in control and DN group rats at 4 weeks after DM. H\&E, PAS and Masson (original magnification $\times 200$ ) showed that, compared with control group, renal fibrosis, glycogen storage, and mesangial matrix expansion were found in $\mathrm{DN}$ group rats. The red arrow shows that glycogen storage, the yellow arrow indicates that mesangial matrix expression. Values are the mean $\pm S D ; n=5, * P<0.05$ compared with the control group at the same time.

Abbreviations: DM, diabetes mellitus; DN, diabetic nephropathy; UACR, urine albumin creatinine ratio; H\&E, hematoxylin-eosin; PAS, periodic Acid-Schiff.

analyzed by HE, PAS and Masson staining. HE staining revealed glomerular hypercellularity in DN group accompanied by basement membrane thickening. Both PAS and Masson staining demonstrated that DN group rats presented obvious pathological changes compared to the normal group, including mesangial matrix expansion and marked glycogen storage (Figure 1G).

According to the biochemical index and histological tests, we confirmed that the DN model was successfully established by the 4 th week after establishing diabetes and there was also marked hyperlipidemia in DN group (Figure 1A-E). After confirming the successful establishment of the DN model, we measured Calpain 10 protein levels in their renal tissues and observed significant upregulation in the kidney biopsies (Figure 2A). The in situ Calpain 10 levels in the renal cortex of the DN rats was upregulated in a time-dependent manner (Figure 2A, D), with significant increase by the 4 th week of modeling, then peaking by the 12 th weeks. In order to evaluate the Calpain 10 level in podocytes within the glomeruli, we 
A

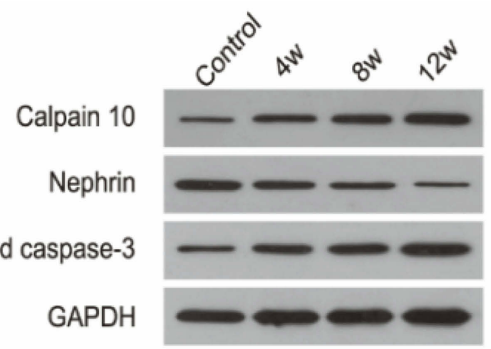

Cleaved caspase-3

GAPDH

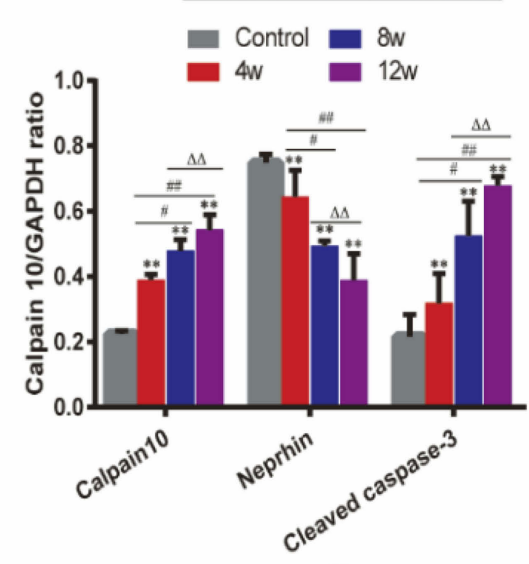

B

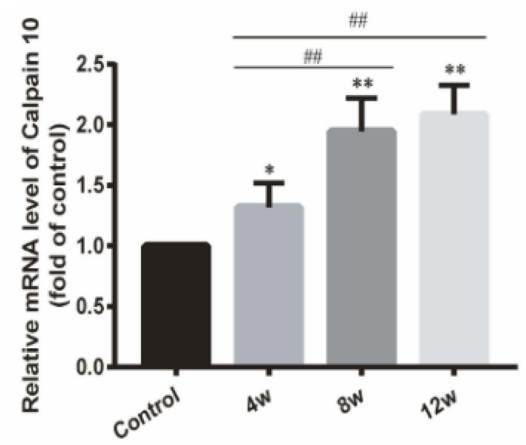

C

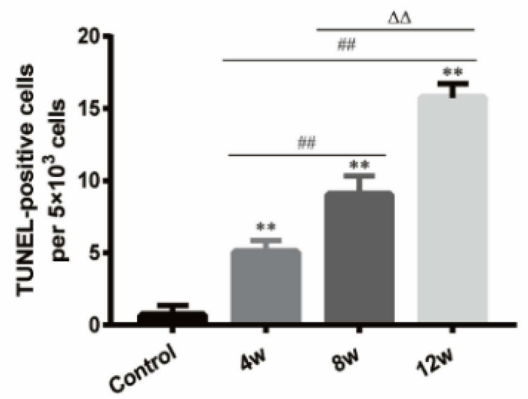

D
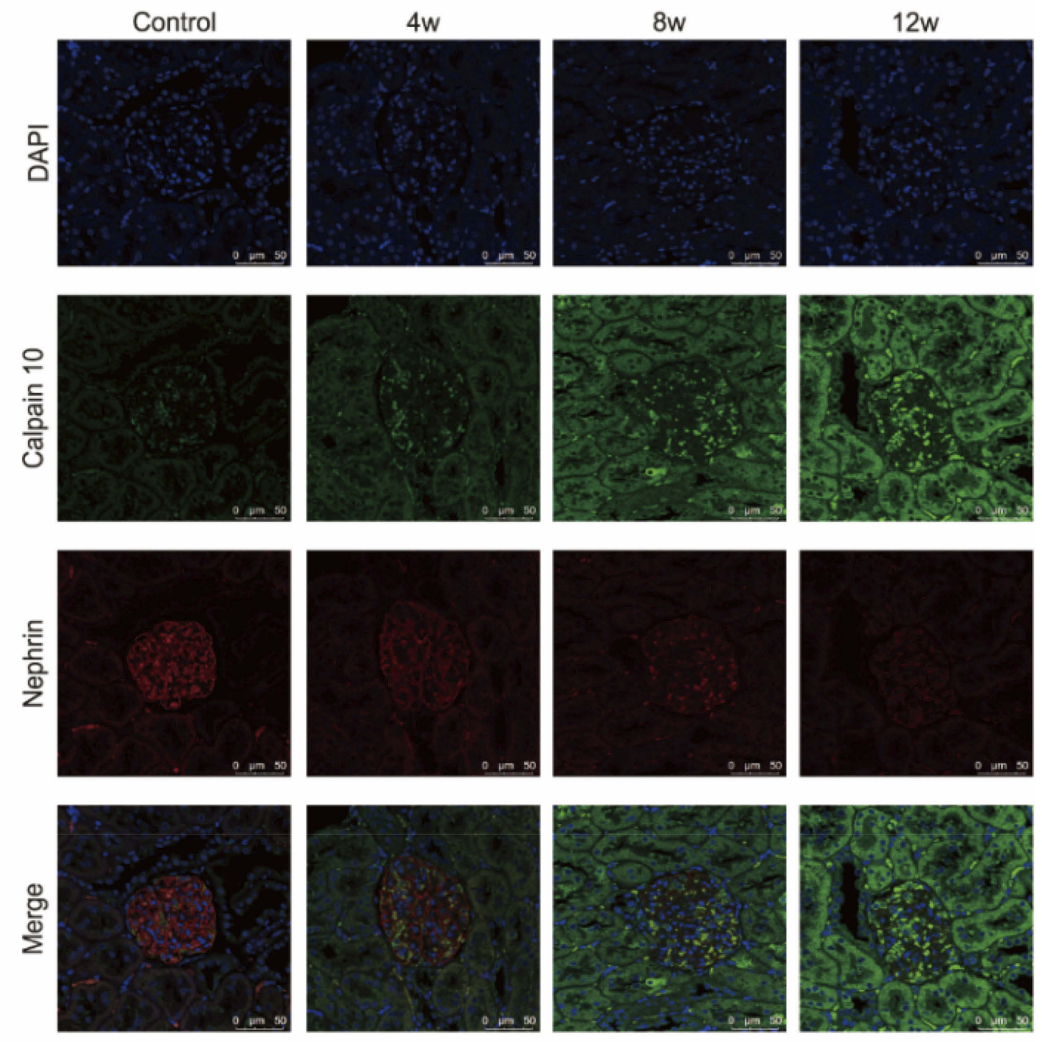

E
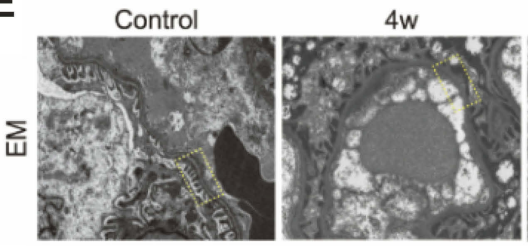

$8 \mathrm{w}$

$12 w$

F

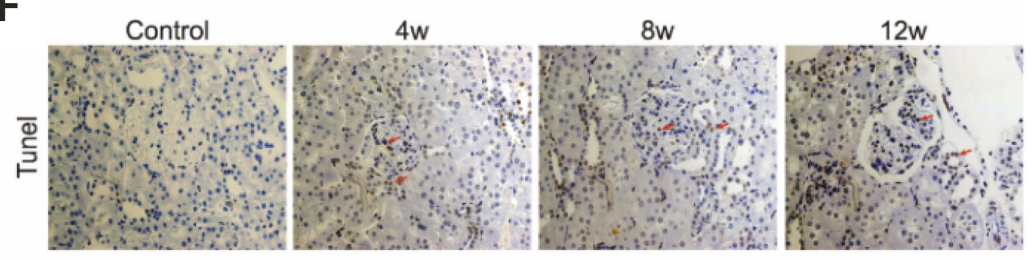

Figure 2 Up-regulation of calpain 10 aggravated renal injury in DN rats. (A) Calpain 10, nephrin and caspase-3 expression in kidney were identified by Western blot analysis. (B) Calpain 10 mRNA expression was tested by Real-time PCR. (C, F) Tissue apoptosis was examined with Tunel positive staining (original magnification $\times 200$ ), arrowheads are showing Tunel-positive cells. (D) Calpain 10 and nephrin expression were identified by immunofluorescence analysis (original magnification $\times 630$ ). (E) EM images showed podocyte foot process fusion, structure disorder and incrassation of glomerular (original magnification $\times 10,000$ ), the dotted yellow boxes indicate the GBM. Values are the mean $\pm S D$; $n=5$, ${ }^{*} P<0.05,{ }^{* * P} P 0.01$ compared with the control group; ${ }^{*} P<0.05,{ }^{\#} P<0.001$ compared with the DN group at 4 weeks; ${ }^{\Delta \Lambda} P<0.01$ compared with the DN group at 8 weeks. Abbreviations: 4/8/I2w, 4, 8, 12 weeks after confirming DM for the DN group; EM, electron microscope.

specifically labeled the podocytes with anti-nephrin antibodies. As shown in Figure 2D, Calpain 10 levels still increased with the decrease in nephrin ${ }^{+}$podocytes in DN rats in a time-dependent manner. Moreover, the intensity of Calpain 10 was still higher in the individual podocytes, indicating that its expression per se was also increased. Compared to the control rats, Calpain 10 mRNA was also upregulated in the DN rats in a time-dependent manner, 
and the mRNA also peaked 12 weeks post-modelling (Figure 2B). Taken together, the expression of Calpain 10 was upregulated in the podocytes of DN rats.

Insulin resistance associated with abnormal lipid metabolism is one of the main characteristics in type 2 diabetes, and dyslipidemia can cause kidney cell damage, accelerate the progress of kidney disease. ${ }^{20,21}$ Palmitate is significantly increased when insulin resistance presents, and is associated with the endoplasmic reticulum stress and apoptosis. $^{22}$ So that we treated the podocytes with palmitate to simulate environmental stimuli for type 2 diabetes.

To determine the optimal PA concentration and incubation time on the podocyte cell line, the cells were exposed to different PA concentrations $(50,100,150 \mu \mathrm{M})$ for 24 , 48 and $72 \mathrm{~h}$; the viability was measured by the CCK-8 assay. As shown in Figure 3A, treatment with $100 \mathrm{Mm}$ PA for $48 \mathrm{~h}$ has the most appropriate cytotoxicity, which induces $30 \%$ decrease of podocytes (also called injured ones). At higher concentrations $(150 \mu \mathrm{M})$ or $100 \mu \mathrm{M}$ for longer inducing time (72 h), the viability of the cells began to decrease significantly; and the treatment with $150 \mu \mathrm{M}$ for $72 \mathrm{~h}$ severely inhibited cell viability. Thus, we simulated the hyperlipidemic conditions of Diabetes Mellitus in vitro by culturing the podocytes under $100 \mu \mathrm{M}$ PA conditions for $48 \mathrm{~h}$ to establish podocyte injury model.

Calpain 10 protein levels started to increase after $24 \mathrm{~h}$ of hyperlipidemia exposure and peaked at $48 \mathrm{~h}$ (Figure 3B). Consistent with this, Calpain-10 mRNA levels were also upregulated under hyperlipidemia conditions (Figure 3C). Therefore, prolonged exposure to PA induced Calpain-10 transcription and translation in the podocytes.

\section{Elevated calpain 10 levels were associated with renal insufficiency and morphology in $\mathrm{DN}$ rats}

We observed a significant negative correlation between renal Calpain 10 levels and renal function in a timedependent manner, as indicated by the significantly higher UACR in the DN rats compared to the control group rats (Figure 1F). In addition, EM were used to observe renal morphology (Figure 2E). The DN group rats showed foot process fusion and structural disorder of podocytes, increased thickness of the glomerular basement membrane (GBM), and the effacement of podocyte foot processes was evident in all the DN rats, especially at the time of 12 weeks (Figure 2E). As with renal dysfunction, the severity of renal morphology was also associated with increased Calpain 10 expression in DN rats.

\section{Hyperlipidemia-induced calpain 10 resulted in podocyte injury in vitro}

To further confirm the role of Calpain 10 in podocyte injury, its expression was inhibited in podocytes using small interfering RNA (siRNA), and the viability was respectively analyzed by CCK- 8 assay. After Calpain 10 knockdown, a significantly higher podocyte viability under hyperlipidemic conditions was shown compared to that under healthy conditions (Figure 3D). Taken together, this suggests Calpain 10 is a crucial factor in hyperlipidemia-induced podocyte injury.

\section{Increase of calpain 10 was correlated with the decrease of nephrin in podocytes}

Significantly lower nephrin protein levels were demonstrated in the renal cortex of DN rats compared to the controls in a time-dependent manner (Figure 2A, D). The degree of decreasing of nephrin protein levels increased with the prolongation of treatment time, and there was a remarkably negative correlation between the expression of nephrin and Calpain 10 (Figure 2D). Consistent with this, hyperlipidemia inhibited nephrin expression in the podocytes in vitro. The level of nephrin in podocytes in vitro also had a negative correlation with the Calpain 10, and was rescued by knocking down Calpain 10(Figure 3F, G). Therefore, the correlation between increased Calpain 10 and renal injury is likely mediated by nephrin inhibition, which can be reversed by blocking Calpain 10 .

\section{High levels of calpain 10 were involved in podocyte apoptosis in vivo and in vitro}

In addition to impaired structure and function, we also observed a significant loss of podocytes in the diabetic kidneys (Figure 2D). We evaluated renal apoptosis in diabetic nephropathy rats by TUNEL staining and measured caspase 3, an apoptosis-related protein. By TUNEL staining, the time-dependent increase of TUNEL-positive cells was observed in the glomeruli and renal tubules of the DN group at various time points (week 4, 8 and 12 after confirming diabetes) (Figure 2C, F). However, unlike the Calpain 10 expression, a significant increase of apoptotic cells was observed by the 8th week. In vitro, Tunnel analysis revealed significantly increased apoptotic podocytes in PA group (Figure 3E, G). Consistent with the TUNEL staining, the 


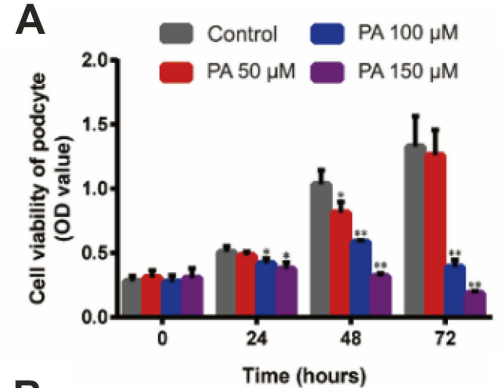

B
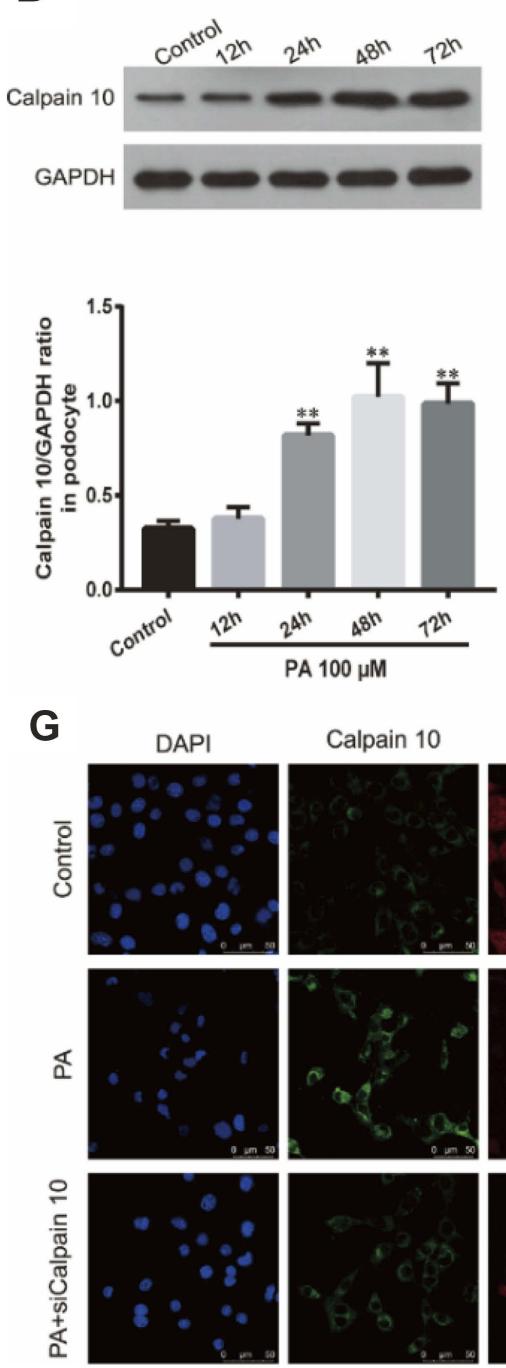

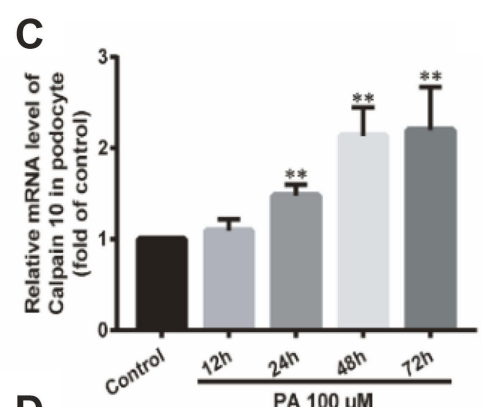

D

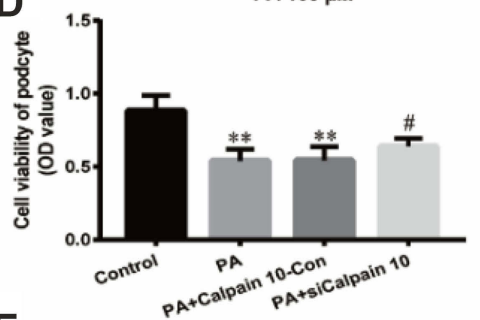

E
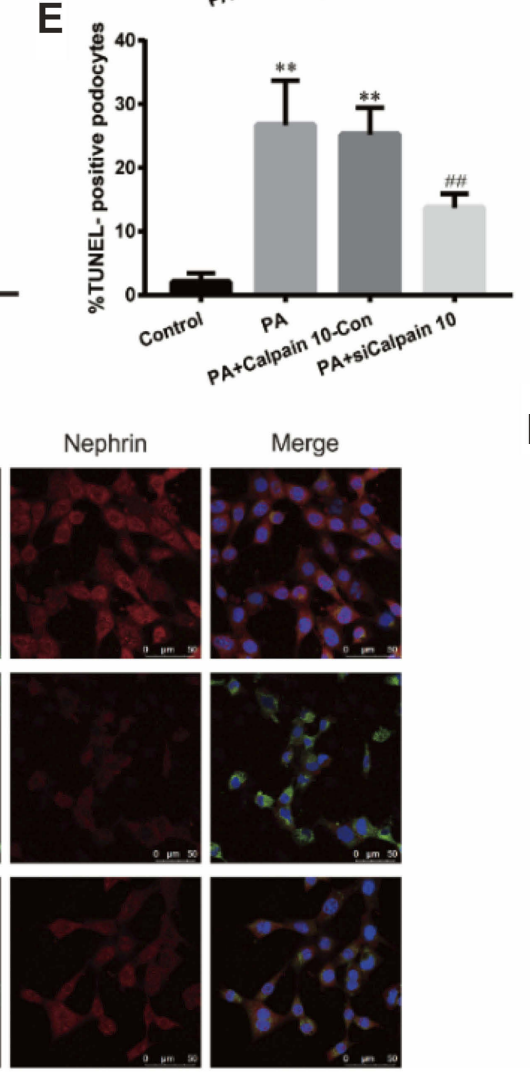

$\mathbf{F}$
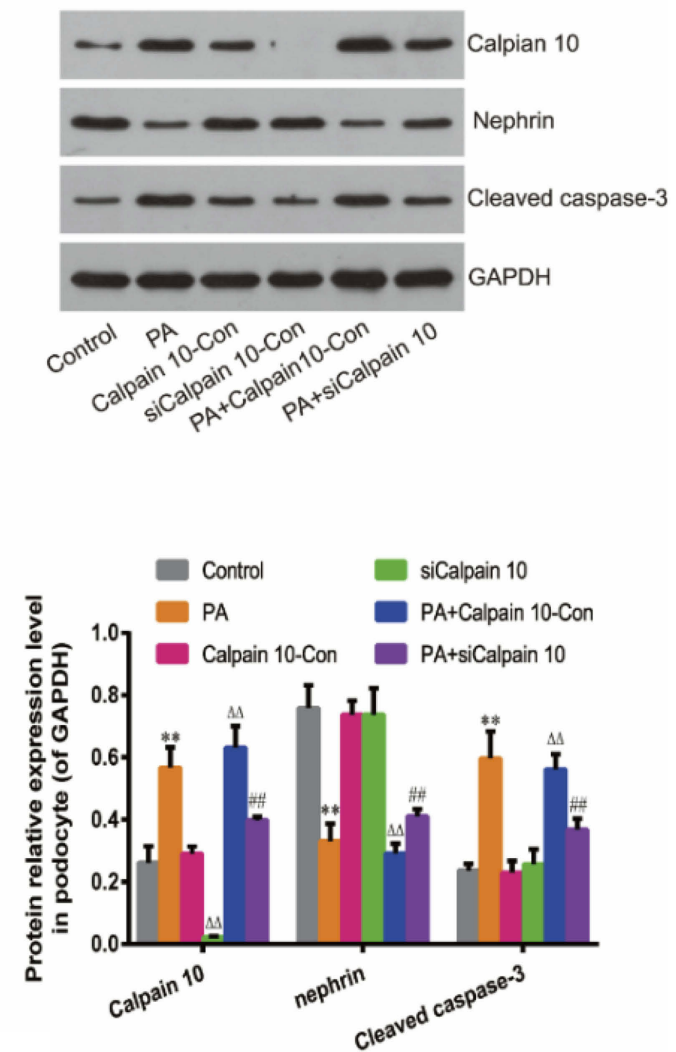

H
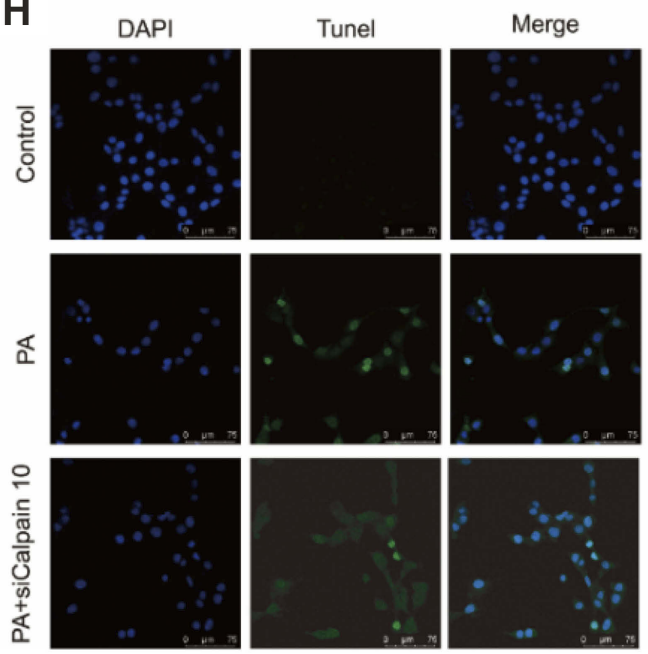

Figure 3 Down-regulation of Calpain 10 protected podocytes and reduced cell apoptosis under hyperlipidemic condition. (A) Effect of PA on podocyte viability. Podocytes were cultured with PA at different concentrations $(50,100,150 \mu \mathrm{M})$ for various times $(24,48,72 \mathrm{~h})$. Relative cell viability was assessed with CCK-8. (B) Calpain 10 expression was identified by Western blot analysis. (C) Calpain 10 mRNA expression was tested by Real-time PCR. (D) Relative cell viability in podocytes treated as indicated was assessed with CCK-8. (E, H) Podocyte apoptosis in Control, PA, PA+siCalpain 10 was examined with Tunel-positive staining (original magnification $\times 400)$. (F) Calpain 10, nephrin and caspase-3 expression in podocytes was identified by Western blot analysis. (G) Immunofluorescent for Calpain 10 and nephrin in podocytes treated as indicated (original magnification $\times 630$ ). Values are the mean $\pm S D ; n=5, * * P<0.01$ compared with the control group; ${ }^{\Delta \Lambda} P<0.01$ compared with the Calpain I0-Con group; ${ }^{\#} P<0.05,{ }^{\#} P<0.01$ compared with the PA+Calpian 10-Con group.

Abbreviations: PA, palmitate; siCalpain 10, the podocytes transfected by the Calpain 10-specific siRNA; Calpian I0-Con, the podocytes transfected by scrambled siRNA.

cleaved caspase 3 levels also revealed increased renal apoptosis in the DN group, as well as in the podocytes cultured in vitro under high PA conditions (Figure 2A, F). These changes in vitro attenuated by Calpain 10 knockdown, suggested that calpain 10 plays an important role in the apoptosis of podocytes in diabetic nephropathy (Figure 3E, F, H). 


\section{Discussion}

Calpains are a family of $\mathrm{Ca} 2+$-activated cysteine proteases that have been involved in the development of diseases, such as type 2 diabetes, Alzheimer's disease, myocardial infarcts, and acute kidney injury). ${ }^{23-26}$ Previous studies found that the expression of pancreatic Calpain 10 was up-regulated in the process of diabetes, which is closely associated with insulin secretion. ${ }^{27,28}$ Calpain 10, a member of the calpain family, is a ubiquitously expressed mitochondrial and cytosolic $\mathrm{Ca} 2+$-regulated cysteine protease, researches have demonstrated that overexpression of Calpain 10 leads to mitochondrial dysfunction and cell death. $^{29}$ Other research showed that Angiotensin II (AngII) induced activation of Calpain 10. ${ }^{24}$ Meanwhile previous studies have found significant increase of renal Angiotensin II (AngII) in DN. ${ }^{30,31}$ In our study, renal Calpain-10 levels increased significantly in the rat model of $\mathrm{DN}$ in a time-dependent manner, peaking 12 weeks after induction of DM. In addition, Calpain-10 expression was positively correlated with renal function, and knocking down calpain-10 significantly protected the podocytes and improved the level of nephrin.

We noted that there is evidence indicated calpain 10 was downregulated in diabetic rats, and contributed to renal cell death. ${ }^{32}$ The conflict between our research and previous studies possibly were attributed to the different ways of modeling. Diabetes was featured by hyperglycemia and hyperlipidemia co-occurred in the DN model of our study. ${ }^{33}$ Previous studies showed that high lipotoxicity was engaged in the occurrence and development of diabetic nephropathy. ${ }^{21,34-37}$ Other researches confirmed the significant calcium overload in diabetic nephropathy. ${ }^{38-41}$ Moreover, calcium overload and lipid metabolites of PA provoked and upregulated calpain 10 which played an essential role in cell apoptosis. ${ }^{27,42}$ Therefore, we speculated that lipotoxicity could be the main reason to lead the upregulated expression of calpain 10 in diabetic nephropathy. Accordingly, we attempted to treat podocytes with PA to build podocyte injury model in vitro.

In addition, previous studies demonstrated that Calpian10 is associated with the cell differentiation and decreases gradually with aging. ${ }^{43}$ It was uncovered that Calpain 10 expressed little in mature lenses of rats, but highly in outer regions of lens, which may be related to cell remodeling in terminal differentiation. However, most of the renal cells (including podocytes) were terminally differentiated cells, ${ }^{44}$ which maybe contribute to the low expression of Calpain 10 in renal tissues of our control group. Marisa D, et al established the diabetic rat model without high-fat diet, so the lipotoxicity might not obviously appeared in their experiment animals. Apart from this, in their study of renal tubular epithelial cells with a certain ability of proliferation, ${ }^{45}$ the expression of Calpain 10 appeared higher in normal condition than ours. However, as the rats ages, the expression of Calpain10 may be down-regulated due to lack of lipotoxicity. In vitro experiments, Marisa D, et al induced renal proximal tubular cells (from female New Zealand White rabbits) with the high glucose instead of PA, which also was a potential reason for the conflict results.

Calpain 10 regulates calcium ion efflux into the cytoplasm from mitochondria and endoplasmic reticulum, and triggers the apoptotic pathway. ${ }^{38}$ Studies show that Calpain 10-induced apoptosis in the cells of the superior renal duct aids in the development of DN. Podocytes are the major cells of the glomerular filtration membrane, and their destruction is the direct cause of proteinuria seen in the early stages of DN. ${ }^{46}$ Therefore, we hypothesized that renal dysfunction in DN is a result of Calpain 10-induced injury and apoptosis in podocytes. Consistent with our hypothesis, renal Calpain 10 levels in the diabetic rats demonstrate a significantly negative correlation with renal function. However, renal dysfunction was observed at a later time point than remarkable Calpain 10 upregulation, which could either be due to a compensatory mechanism in the kidneys, or due to sub-optimal Calpain 10 levels in the podocytes. Nephrin is the major protein of the glomerular filtration membrane, which plays a key role in regulating and stabilizing the integrity of the glomerular filtration barrier and renal function. ${ }^{47}$ In vivo studies have shown that the increased expression of Calpain 10 decreased that of nephrin in glomerular podocytes, indicating that Calpain 10 is involved in podocyte injury in DN. Consistent with previous findings, high PA levels in vitro decreased podocyte activity, downregulated nephrin protein levels, and were concomitant with increased levels of Calpain 10. Therefore, nephrin levels were significantly reduced in the podocytes both in vivo and in vitro, indicating that podocyte injury and dysfunction are initiated early on by Calpain 10 upregulation. ${ }^{48}$ Knockdown of Calpain10 significantly reversed the above changes, which underscored the role of Calpain 10 in podocyte injury in DN.

Studies showed that the pathogenesis of DN was accompanied by podocyte apoptosis, ${ }^{49-52}$ which we also observed in the DN group rats. Although scattered apoptotic cells 
were seen in the glomeruli after 4 weeks of modelling, it increased drastically after 8 weeks. This was consistent with considerable podocyte numbers, as determined by the expression of nephrin. Furthermore, apoptosis in the cultured podocytes was observed within $48 \mathrm{hrs}$ of high PA stimulation. Previous study showed that Calpain-10 which is a mitochondrial and cytosolic $\mathrm{Ca}^{2+}$-regulated cysteine protease is associated with apoptosis. ${ }^{7,8}$ Therefore, we speculated that the decrease in podocytes during diabetes is a direct result of Calpain 10-induced apoptosis, and we did find that blocking Calpain 10 can reduce the apoptosis index of DN kidney and cultured podocytes. In conclusion, Calapin-10 is a promising therapeutic target in DN and warrants further study.

\section{Conclusion}

Calpain 10 is upregulated under hyperlipidemic conditions, and results in podocyte injury and apoptosis, ultimately correlated with decreased renal function. Inhibition of Calpain 10 improves nephrin level in podocytes and protects the podocytes against apoptosis, indicating its potential as a therapeutic target in $\mathrm{DN}$.

\section{Acknowledgment}

This study was supported by grant from the Major National Basic Research Program of China (973 Program, no.2012CB518602).

\section{Disclosure}

The authors report no conflicts of interest in this work.

\section{References}

1. Fried LF, Emanuele N, Zhang JH, et al. Combined angiotensin inhibition for the treatment of diabetic nephropathy. N Engl J Med. 2013; 369(20):1892-1903 doi:10.1056/NEJMoa1303154

2. Hakroush S, Cebulla A, Schaldecker T, Behr D, Mundel P, Weins A Extensive podocyte loss triggers a rapid parietal epithelial cell response. J Am Soc Nephrol. 2014;25(5):927-938. doi:10.1681/ ASN.2013070687

3. Wang ZS, Xiong F, Xie XH, Chen D, Pan JH, Cheng L Astragaloside IV attenuates proteinuria in streptozotocin-induced diabetic nephropathy via the inhibition of endoplasmic reticulum stress. BMC Nephrol 2015;16: 44

4. Mallipattu SK, He JC The podocyte as a direct target for treatment of glomerular disease? Am J Physiol Renal Physiol. 2016;311(1): F46-F51

5. Yasuda-Yamahara M, Kume S, Tagawa A, Maegawa H, Uzu T Emerging role of podocyte autophagy in the progression of diabetic nephropathy. Autophagy. 2015;11(12):2385-2386

6. Ravindran S, Kuruvilla V, Wilbur K, et al. Nephroprotective effects of metformin in diabetic nephropathy. J Cell Physiol. 2017;232(4):731742 doi:10.1002/jcp.v232.4.

7. Goll DE, Thompson VF, Li H, et al. The calpain system. Physiol Rev 2003;83:731-801
8. Huang Y, Wang KK The calpain family and human disease. Trends Mol Med 2001;7:355-362

9. Hanis CL, Boerwinkle E, Chakraborty R, et al. A genome-wide search for human non-insulin-dependent (type 2) diabetes genes reveals a major susceptibility locus on chromosome 2. Nat Genet 1996;13:161-166

10. Harris F, Biswas S, Singh J, et al. Calpains and their multiple roles in diabetes mellitus. Ann N Y Acad Sci 2006;1084:452-480

11. Cai X, Wang L, Wang X, Hou F miR-124a enhances therapeutic effects of bone marrow stromal cells transplant on diabetic nephropathy-related epithelial-to-mesenchymal transition and fibrosis. J Cell Biochem. 2019. doi:10.1002/jcb.29170

12. Tian N, Gao Y, Wang X, et al. Emodin mitigates podocytes apoptosis induced by endoplasmic reticulum stress through the inhibition of the PERK pathway in diabetic nephropathy. Drug Des Devel Ther. 2018;12:2195-2211. doi:10.2147/DDDT.S167405

13. Singhal S, Rathore AS, Lohar V, Dave R, Dave J Pharmacological evaluation of "sugar remedy," a polyherbal formulation, on streptozotocin-induced diabetic mellitus in rats. $J$ Tradit Complement Med. 2014;4(3):189-195. doi:10.4103/2225-4110.127800

14. Kuhad A, Sachdeva AK, Chopra K Attenuation of renoinflammatory cascade in experimental model of diabetic nephropathy by sesamol. $J$ Agric Food Chem. 2009;57(14):6123-6128. doi:10.1021/jf901388g

15. Soliman GA, Saeedan AS, Abdel-Rahman RF, et al. Olive leaves extract attenuates type II diabetes mellitus-induced testicular damage in rats: molecular and biochemical study. Saudi Pharm J. 2019;27 (3):326-340. doi:10.1016/j.jsps.2018.11.015

16. Vitlov Uljević M, Starčević K, Mašek T, et al. Dietary DHA/EPA supplementation ameliorates diabetic nephropathy by protecting from distal tubular cell damage. Cell Tissue Res. 2019. doi:10.1007/ s00441-019-03058-y

17. Abdelrahman AM, Al Suleimani Y, Shalaby A, Ashique M, Manoj P, Ali BH Effect of tocilizumab, an interleukin-6 inhibitor, on early stage streptozotocin-induced diabetic nephropathy in rats. Naunyn Schmiedebergs Arch Pharmacol. 2019;392(8):1005-1013. doi:10.1007/s00210-019-01655-w

18. Qin Q, Lin N, Huang H, et al. Ginsenoside Rg1 ameliorates cardiac oxidative stress and inflammation in streptozotocin-induced diabetic rats. Diabetes Metab Syndr Obes. 2019;12:1091-1103. doi:10.2147/ DMSO.S208989

19. Huang Y, Xu J, Wu X, et al. High expression of complement components in the kidneys of type 2 diabetic rats with diabetic nephropathy. Front Endocrinol (Lausanne). 2019;10:459. doi:10.3389/ fendo.2019.00459

20. Druilhet RE, Overturf ML, Kirkendall WM Structure of neutral glycerides and phosphoglycerides of human kidney. Nt J Biochem, 1975, 6(12):893-901. doi:10.1016/0020-711X(75)90010-5

21. Kim Y, Hwang SD, Lim JH, et al. Attenuated lymphatic proliferation ameliorates diabetic nephropathy and high-fat diet-induced renal lipotoxicity. Sci Rep. 2019;9(1):1994. doi:10.1038/s41598-01838250-7

22. Lennon R, Pons D, Sabin MA, et al. Saturated fatty acids induce insulin resistance in human podocytes: implicationsfor diabetic nephropathy. Nephrol Dial Transplant, 2009,24(11):3288-3296. doi:10.1093/ndt/gfp302

23. Chatterjee PK, Todorovic Z, Sivarajah A, et al. Inhibitors of calpain activation (PD150606 and E-64) and renal ischemia-reperfusion injury. Biochem Pharmacol. 2005;69:1121-1131. doi:10.1016/j. bcp. 2005.01 .003

24. Seremwe M, Schnellmann RG, Bollag WB Calpain-10 activity underlies angiotensin II-induced aldosterone production in an adrenal glomerulosa cell model. Endocrinology. 2015;156(6):2138-2149. doi:10.1210/en.2014-1866

25. Suzuki K, Hata S, Kawabata Y, Sorimachi H Structure, activation, and biology of calpain. Diabetes. 2004;53:S12-S18. doi:10.2337/ diabetes.53.6.1467 
26. Chatterjee PK, Brown PAJ, Cuzzocrea S, et al. Calpain inhibitor-1 reduces renal ischemia/reperfusion injury in the rat. Kidney Int. 2001; 59:2073-2083. doi:10.1046/j.1523-1755.2001.00722.x

27. Marshall C, Hitman GA, Partridge CJ, et al. Evidence that an isoform of calpain-10 is a regulator of exocytosis in pancreatic beta-cells. $\mathrm{Mol}$ Endocrinol. 2005;19(1):213-224. doi:10.1210/me.2004-0064

28. Hatta T, Iemura SI, Ohishi $T$, et al. Calpain-10 regulates actin dynamics by proteolysis of microtubule-associated protein 1B. Sci Rep. 2018;8(1):16756. doi:10.1038/s41598-018-35204-x

29. Arrington DD, Van Vleet TR, Schnellmann RG Calpain 10: a mitochondrial calpain and its role in calcium-induced mitochondrial dysfunction. Am J Physiol Cell Physiol. 2006;291:C1159-C1171. doi:10.1152/ajpcell.00116.2006

30. Kang JS, Lee SJ, Lee JH, et al. Angiotensin II-mediated MYH9 downregulation causes structural and functional podocyte injury in diabetic kidney disease. Sci Rep. 2019;9(1):7679. doi:10.1038/ s41598-019-44194-3

31. Makary S, Abdo M, Hassan WA, Tawfik MK Angiotensin blockade attenuates diabetic nephropathy in hypogonadal adult male rats. Can J Physiol Pharmacol. 2019;97(8):708-720. doi:10.1139/cjpp-2018-0572

32. Covington MD, Schnellmann RG Chronic high glucose downregulates mitochondrial calpain 10 and contributes to renal cell death and diabetes-induced renal injury. Kidney Int. 2012;81(4):391-400. doi:10.1038/ki.2011.356

33. Mousum SA, Ahmed S, Gawali B, Kwatra M, Ahmed A, Lahkar M Nyctanthes arbor-tristis leaf extract ameliorates hyperlipidemia- and hyperglycemia-associated nephrotoxicity by improving anti-oxidant and anti-inflammatory status in high-fat diet-streptozotocin-induced diabetic rats. Inflammopharmacology. 2018;26(6):1415-1428. doi:10.1007/ s10787-018-0497-6

34. Choi SR, Lim JH, Kim MY, et al. Adiponectin receptor agonist AdipoRon decreased ceramide, and lipotoxicity, and ameliorated diabetic nephropathy. Metabolism. 2018;85:348-360. doi:10.1016/j. metabol.2018.02.004

35. Lee ES, Kwon MH, Kim HM, et al. Dibenzoylmethane ameliorates lipid-induced inflammation and oxidative injury in diabetic nephropathy. J Endocrinol. 2019;240(2):169-179. doi:10.1530/JOE-18-0206

36. Hwang SD, Song JH, Kim Y, et al. Inhibition of lymphatic proliferation by the selective VEGFR-3 inhibitor SAR131675 ameliorates diabetic nephropathy in $\mathrm{db} / \mathrm{db}$ mice. Cell Death Dis. 2019;10 (3):219. doi:10.1038/s41419-019-1300-3

37. Li X, Zhang T, Geng J, et al. Advanced oxidation protein products promote lipotoxicity and tubulointerstitial fibrosis via CD36/ $\beta$-catenin pathway in diabetic nephropathy. Antioxid Redox Signal. 2019;31 (7):521-538. doi:10.1089/ars.2018.7634

38. Chen W, Xiang H, Chen R, et al. S1PR2 antagonist ameliorate high glucose-induced fission and dysfunction of mitochondria in HRGECs via regulating ROCK1. BMC Nephrol. 2019;20(1):135. doi:10.1186/ s12882-018-1145-5
39. Ansari M, Kurian GA Hydrogen sulfide preconditioning could ameliorate reperfusion associated injury in diabetic cardiomyopathy rat heart through preservation of mitochondria. Biochimie. 2019;158: 208-216. doi:10.1016/j.biochi.2019.01.011

40. Yang F, Qin Y, Wang Y, et al. LncRNA KCNQ1OT1 mediates pyroptosis in diabetic cardiomyopathy. Cell Physiol Biochem. 2018;50(4):1230-1244. doi:10.1159/000494576

41. Wang J, Tang Z, Zhang Y, et al. Matrine alleviates AGEs- induced cardiac dysfunctions by attenuating calcium overload via reducing ryanodine receptor 2 activity. Eur J Pharmacol. 2019;842:118-124. doi:10.1016/j.ejphar.2018.10.010

42. Johnson JD, Han Z, Otani K, et al. RyR2 and calpain-10 delineate a novel apoptosis pathway in pancreatic islets. J Biol Chem. 2004;279 (23):24794-24802. doi:10.1074/jbc.M401216200

43. Ma H, Fukiage C, Kim YH, et al. Characterization and expression of calpain 10. A novel ubiquitous calpain with nuclear localization. J Biol Chem 2001;276(30):28525-28531. doi:10.1074/jbc.M100603200

44. Mundel P, Reiser J, Kriz W Induction of differentiation in cultured rat and human podocytes. J Am Soc Nephrol. 1997;8(5):697-705.

45. Morizane R, Miyoshi T, Bonventre JV Concise review: kidney generation with human pluripotent stem cells. Stem Cells. 2017;35 (11):2209-2217. doi:10.1002/stem.2699

46. Xu J, Zheng S, Patricia M, et al. Diabetes induced changes in podocyte morphology and gene expression evaluated using GFP transgenic podocytes. Int J Biol Sci. 2016,12,210-218. doi:10.7150/ ijbs. 13057

47. Verma R, Venkatareddy M, Kalinowski A, et al. Nephrin is necessary for podocyte recovery following injury in an adult mature glomerulus. PLoS One. 2018;13(6):e0198013. doi:10.1371/journal. pone. 0198013

48. Huang CJ, Gurlo T, Haataja L, et al. Calcium-activated calpain-2 is a mediator of beta cell dysfunction and apoptosis in type 2 diabetes. $J$ Biol Chem, 2010, 285: 339-348. doi:10.1074/jbc.M109.024190

49. Huang G, Zou B, Lv J, et al. Notoginsenoside R1 attenuates glucoseinduced podocyte injury via the inhibition of apoptosis and the activation of autophagy through the PI3K/Akt/mTOR signaling pathway. Int $J$ Mol Med. 2017;39(3):559-568. doi:10.3892/ijmm.2017.2864

50. $\mathrm{Xu} \mathrm{S,} \mathrm{Nam} \mathrm{SM,} \mathrm{Kim} \mathrm{JH,} \mathrm{et} \mathrm{al.} \mathrm{Palmitate} \mathrm{induces} \mathrm{ER} \mathrm{calcium}$ depletion and apoptosis in mouse podocytes subsequent to mitochondrial oxidative stress. Cell Death Dis. 2015 19;6: e1976. doi:10.1038/ cddis.2015.331

51. Jin Y, Liu S, Ma Q, Xiao D, Chen L Berberine enhances the AMPK activation and autophagy and mitigates high glucose-induced apoptosis of mouse podocytes. Eur J Pharmacol. 2017;794:106-114. doi:10.1016/j.ejphar.2016.11.037

52. Dai H, Liu Q, Liu B Research progress on mechanism of podocyte depletion in diabetic nephropathy. $J$ Diabetes Res. 2017; 2017:2615286. doi:10.1155/2017/2615286

Diabetes, Metabolic Syndrome and Obesity: Targets and Therapy

\section{Publish your work in this journal}

Diabetes, Metabolic Syndrome and Obesity: Targets and Therapy is an international, peer-reviewed open-access journal committed to the rapid publication of the latest laboratory and clinical findings in the fields of diabetes, metabolic syndrome and obesity research. Original research, review, case reports, hypothesis formation, expert opinion and commentaries are all considered for publication. The manuscript management system is completely online and includes a very quick and fair peer-review system, which is all easy to use. Visit http://www.dovepress.com/testimonials.php to read real quotes from published authors. 